\title{
Modular Implementation of a Hybrid DSMC-NS Algorithm for Hypersonic Non-Equilibrium Flows
}

\author{
Thomas E. Schwartzentruber*, Leonardo C. Scalabrin ${ }^{\dagger}$ and Iain D. Boyd ${ }^{\ddagger}$ \\ Department of Aerospace Engineering, University of Michigan, Ann Arbor, MI 48109
}

\begin{abstract}
The implementation of a modular particle-continuum (MPC) numerical method is presented which solves the Navier-Stokes (NS) equations in regions of near-equilibrium and uses the direct simulation Monte Carlo (DSMC) method where the flow is in non-equilibrium. A mesh refinement procedure is described which allows the NS equations to be solved on a relatively coarse mesh using large implicit time-steps while the DSMC method is used in localized regions on a refined mesh. The MPC method accommodates a novel modular implementation which combines existing, state-of-the-art, DSMC and NS source codes, virtually un-modified, into a single modular code. Hypersonic flow over a 2D cylinder at various Mach numbers is simulated using DSMC, NS, and MPC methods and results are compared for accuracy. Added mesh refinement capability increases the efficiency of the MPC method which is able to reproduce DSMC flow field results for simple transitional flows up to 3 times faster while requiring half the memory. When applied to a mainly continuum flow, the MPC method reproduces non-equilibrium DSMC results in the near-wake region approximately 10 times faster than full DSMC simulation while using only $8 \%$ of the memory.
\end{abstract}

\section{Introduction}

$\mathrm{H}$ YPERSONIC vehicles can experience both high-speed rarefied conditions as well as lower-speed continuum conditions during different portions of their trajectories. When it comes to numerical simulation of the aerothermodynamics around a vehicle in both of these flow regimes, mature numerical methods already exist. For example, the direct simulation Monte Carlo (DSMC) method $^{1}$ is an accurate and efficient method for simulation in the rarefied regime. At lower altitudes where the DSMC method becomes extremely computationally demanding, the continuum Navier-Stokes (NS) equations accurately model the flow and can be solved efficiently using algorithms from Computational Fluid Dynamics (CFD). However, difficulty arises for numerical simulation at intermediate altitudes where the flow is mainly continuum except for localized non-equilibrium regions inside shock waves, boundary layers, wakes, and over sharp leading edges. For example, in hypersonic blunt-body flows, the majority of the flow may be continuum except for the near-wake and possibly regions of the boundary layer where the NS equations are no longer an accurate model. ${ }^{2}$ For such flows, DSMC is needed to accurately predict conditions in the near-wake but becomes computationally intractable in the fore-body region. This is due to the disparate length and time scales required for particle simulation of both the wake and fore-body regions. By its very nature, a molecular simulation must be performed at high spatial and temporal resolution. Specifically the cell size must remain smaller than the local mean-free-path and the time step must remain less than the local mean-free-time. Thus, if large continuum portions of the flow are simulated using DSMC, substantial computational resources are required.

For this reason various researchers have studied a "zonally decoupled" DSMC-NS simulation technique. ${ }^{3-5}$ Here, a NS solution of the fore-body region is performed and the data along the exit plane is extracted and used as the inflow conditions for a separate DSMC simulation of only the wake region. However, in many blunt-body flows of interest, the majority of the wake region may also lie well within the continuum regime and only very localized regions may exhibit non-equilibrium effects. For example, recent CFD simulations of the Apollo AS-202 command module suggest non-equilibrium flow is likely present near the shoulder and rear apex of the capsule during the high altitude "skip" phase of re-entry. ${ }^{6}$ These regions are very localized, but may explain the over-prediction in heating rates at these points, made by the CFD simulations. In order to limit the DSMC method to only such localized regions of

*Graduate Student, Student Member AIAA. Email: schwartt@umich.edu.

${ }^{\dagger}$ Graduate Student, Student Member AIAA. Email: 1scalabr@umich.edu.

*Professor, Associate Fellow AIAA. Email: iainboyd@umich.edu. 
non-equilibrium flow, various researchers have proposed hybrid numerical methods which adaptively re-position the DSMC-NS interfaces during the simulation. ${ }^{7-10}$ Wijesinghe et al. embed the DSMC method in the finest level of an adaptive mesh and algorithmic refinement (AMAR) scheme for the Euler equations. ${ }^{11}$ Such an approach is able to limit the use of DSMC to non-equilibrium regions and at the same time is able to effectively separate the disparate length scales found in multi-scale flows. These methods then couple particle and continuum regions by transferring information across their interface using a variety of procedures. Reference 12 presents a discussion of the major considerations involved as well as a summary of published work on such coupled schemes. The modular particlecontinuum (MPC) method discussed in this article was first developed and tested on 1D normal shock waves ${ }^{13}$ and has recently been modified and extended for 2D flows. ${ }^{14}$ In this previous work, the MPC method successfully limited the use of DSMC to only the shock, boundary layer, and near-wake regions and solved the NS equations in the remainder of the flow field. The MPC method was verified to reproduce the local velocity distributions, flow fields and surface properties calculated by full DSMC simulations. In simulating only a portion of the flow using DSMC, the MPC method was able to use fewer particles to reproduce full DSMC results up to 2.8 times faster. ${ }^{14}$ However, this initial research used the same mesh (refined to the mean-free-path) for both DSMC and NS regions. Thus the NS solver was highly inefficient and as a result, introduced significant computational overhead to the MPC simulations. ${ }^{14}$ In addition, since the mesh density was restricted to the mean-free-path everywhere, the simulation of lower Knudsen number flows $(K n<0.01)$ within practical time and memory limits was not possible on a scalar computer.

In this article, a mesh refinement algorithm is outlined which is naturally accommodated by the existing MPC method. For steady-state flow fields containing both rarefied and continuum regions, the MPC method loosely couples existing DSMC and NS codes which now operate on different meshes with different time steps. This allows for the accurate and efficient resolution of the disparate length and time scales involved in hypersonic flows. Next, the modular implementation of the MPC method is completely described. This novel implementation enables state-of-theart, well validated, DSMC and NS source code to be used virtually un-modified as modules within the hybrid code. There are many benefits to such an approach. The initial development time for a hybrid code is substantially reduced and further updates to either module can be rapidly incorporated. Large source codes which have been extensively de-bugged and optimized remain intact and can therefore be used with confidence in the hybrid code. Finally, the accuracy of the complex physical modelling employed by the existing DSMC and NS modules is well understood in each of their respective flow regimes. Hybrid simulations of previous base-line cases ${ }^{14}$ are repeated using the added mesh refinement capability and the reduction in overhead of the MPC method is analyzed. The MPC method is then applied to a lower Knudsen number $(K n=0.002)$ blunt-body flow and the results are compared with a computationally intensive full DSMC simulation.

\section{DSMC and Navier-Stokes Modules}

Particle regions are simulated using MONACO, ${ }^{15}$ a general, cell-based implementation of the DSMC method. ${ }^{1}$ The variable hard sphere (VHS) collision model is employed which results in the following macroscopic viscosity model: ${ }^{1}$

$$
\mu=\mu_{r e f}\left(\frac{T}{T_{r e f}}\right)^{\omega}, \quad \mu_{r e f}=\frac{15 \sqrt{\pi m k T_{r e f}}}{2 \pi d_{r e f}^{2}(5-2 \omega)(7-2 \omega)} .
$$

All numerical results presented in this article are for diatomic nitrogen with a reference diameter of $d_{\text {ref }}=4.17 \times$ $10^{-10} \mathrm{~m}$ at $T_{\text {ref }}=273 \mathrm{~K}$. The power law exponent, $\omega$, is set equal to $0.75, \mathrm{~m}$ is the mass of an $N_{2}$ molecule, and $k$ is the Boltzmann constant. MONACO employs the variable rotational energy exchange probability model of Boyd ${ }^{16}$ where the reference temperature for rotational energy exchange is specified as $91.5 \mathrm{~K}$ and the maximum rotational collision number as 18.1. Energy transfer to vibrational modes is not considered.

Continuum regions are simulated using the LeMANS code. ${ }^{17}$ For the results of this article, it is assumed that rotational and translational energy modes can be described by a single temperature $T$ in continuum regions. The vibrational energy mode is not considered. The resulting governing equations are the well-known, 2D laminar, compressible, Navier-Stokes (NS) equations. The viscosity in the NS solver is modelled using Eq. 1 in order to match exactly the viscosity model used in DSMC. LeMANS solves this set of equations using a finite-volume formulation. The inviscid fluxes between the mesh volumes are discretized using a modified form of the Steger-Warming Flux Vector Splitting ${ }^{18}$ which is less dissipative than the original form. The modified form is thus adequate to calculate boundary layers and the scheme switches back to the original form of Steger-Warming near shock waves. The viscous terms are calculated using the values of properties at the cell centers and at the nodes. The time integration is performed using a point-implicit method. Specific details of the numerical method are contained in Ref. 17. 


\section{Flow Conditions}

The same baseline flow conditions presented in Ref. 14 are revisited in the current study. Specifically, highspeed flow about a 2D cylinder at Mach 3, 6, and 12, is simulated. The free-stream gas is $N_{2}$ with a number density $n=1.61 \times 10^{21} 1 / \mathrm{m}^{3}$ and $T=217.45 \mathrm{~K}$. This corresponds to an altitude of $70 \mathrm{~km}$, where $p=4.83 \mathrm{~Pa}$ and $\rho=$ $7.48 \times 10^{-5} \mathrm{~kg} / \mathrm{m}^{3}$. The diameter of the cylinder is $8.0 \mathrm{~cm}$ resulting in a global Knudsen number of 0.01 . Only the free-stream velocities differ for each case and are set to achieve the desired Mach numbers. The cylinder wall temperature is set to $300 \mathrm{~K}, 500 \mathrm{~K}$, and $1000 \mathrm{~K}$ for the Mach 3, 6, and 12 cases respectively. The DSMC module assumes diffuse reflection and full thermal accommodation at the cylinder wall whereas the NS solver employs no-slip conditions. Previous hybrid results for these flow conditions did not utilize mesh refinement and thus used the same mesh for all NS, DSMC, and MPC simulations. For each case, the local cell size was verified to be less than the mean-free-path throughout the domain and the resulting mesh contained 180,000 cells. ${ }^{14}$ These previous results are herein referred to as cases M3, M6, and M12. For the same flow conditions, this article presents MPC simulations with mesh refinement which are denoted as cases $\mathbf{M} \mathbf{3}_{\mathbf{R}}, \mathbf{M} \mathbf{6}_{\mathbf{R}}$, and $\mathbf{M} 1 \mathbf{2}_{\mathbf{R}}$. For these cases the NS module of the hybrid code operates on a much coarser mesh containing 30,000 cells and the DSMC regions are refined as needed. The mesh density required by the NS module must be determined by employing a standard grid-convergence study while obtaining the initial NS solution. For all flow conditions, the NS solution obtained using the 30,000 cell mesh was verified to reproduce the previous solution obtained on the 180,000 cell mesh. An additional case, denoted as $\mathbf{M 1 2}_{\mathbf{R}-\mathbf{2}}$, raises the free-stream density of case $\mathbf{M 1 2} \mathbf{R}_{\mathbf{R}}$ by a factor of 5, resulting in a blunt-body flow with a global Knudsen number of 0.002 .

\section{Modular Implementation}

\section{A. Mesh Refinement}

The MPC method begins with a pure NS solution obtained on a structured mesh designed for the continuum equations. Although research into un-structured meshes for hypersonic flows is on-going, structured meshes are currently required to properly resolve the large gradients found in hypersonic shock waves and boundary layers. ${ }^{19}$ Using the initial NS solution, a measure of continuum breakdown is calculated in each cell. The parameter used by the MPC method is the gradient-length Knudsen number ${ }^{20}$ given by

$$
K n_{G L}=\max \left(\frac{\lambda}{Q}|\nabla Q|\right)
$$

where the maximum is taken over the flow quantities $(Q)$ of interest; density $(\rho)$, temperature $(T)$, and velocity magnitude $(|V|)$. The local mean-free-path is calculated in each cell of the continuum solution as

$$
\lambda=\frac{2(5-2 \omega)(7-2 \omega)}{15} \sqrt{\frac{m}{2 \pi k T}}\left(\frac{\mu}{\rho}\right)
$$

where $\rho$ is the local density and $\mu$ is the local viscosity. This expression for $\lambda$ is consistent with the VHS viscosity model from Eq. 1 and all variables have the same values as previously outlined. In addition to its use in determining continuum breakdown, $\lambda$ is also a measure of how much refinement is necessary in the DSMC regions. Figure 1 plots contours of $\lambda$ from the initial NS solution used for the $\mathbf{M} \mathbf{6}_{\mathbf{R}}$ case. Here, the bow shock is clearly defined by contour number 2. Also, Fig. 1 reveals that $\lambda$ is more than an order of magnitude smaller in the fore-body stagnation region compared to the near wake region. This cell-centered value of $\lambda$ is then compared to the length of each cell face in order to determine the refinement required in each NS cell. In order for an accurate DSMC simulation, the refined cell size should be less than the local value of $\lambda$. This restriction is most important in the direction of the flow gradient, however, it is less important in the direction normal to this gradient. The mesh refinement procedure used in this article relaxes the cell size in the direction normal to the flow gradient to $4 \lambda$. This is accomplished by setting the refinement for each cell face as

$$
r_{f}=l_{f} \times\left[F \lambda\left(3\left|\hat{n}_{f} \cdot \frac{\nabla Q}{|\nabla Q|}\right|+1\right)\right]^{-1} .
$$

Here, $l_{f}$ is the length of the cell face which is compared to a multiple of the mean-free-path, $\lambda$. The dot product between the face unit normal vector, $\hat{n}_{f}$, and the unit gradient vector determines the alignment of the cell face with the direction of the gradient. If the cell face is perfectly aligned with the flow gradient (which means $\hat{n}_{f}$ is normal to 


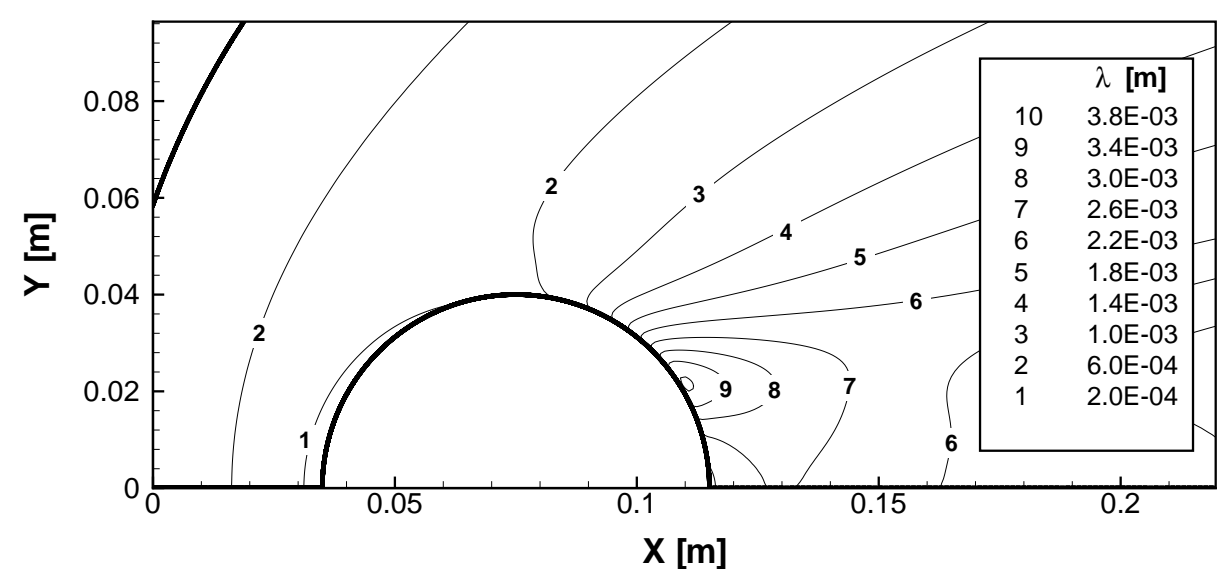

Figure 1. Local mean-free-path for case $M 6_{\mathbf{R}}$.

the flow gradient), the dot product in Eq. 4 is zero, and the refinement is scaled to $F \lambda$. Whereas, if the cell face is perpendicular to the flow gradient (meaning $\hat{n}_{f}$ is parallel to the gradient vector), the refinement is relaxed to $4 F \lambda$. Since the refinement is required because of continuum breakdown, for consistency, the gradient in Eq. 4 is calculated using the same flow quantity ( $\rho, T$, or $|V|$ ) which resulted in the maximum value of $K n_{G L}$ in that cell (Eq. 2). Finally, the factor $F$ in Eq. 4 allows scaling of the entire mesh by any fraction (or multiple) of the local mean-free-path. The entire expression is rounded to the nearest integer to obtain $r_{f}$. Since opposite faces in a quadrilateral cell must be refined equally, each NS cell will contain two integers $\left(r_{1}\right.$ and $\left.r_{2}\right)$ specifying the minimum required refinement in orthogonal directions. The current DSMC module also requires that each cell face border only one neighboring cell. As a result, the refinement can not be set arbitrarily in each cell but must be consistent with neighboring cells. A simple algorithm is employed which ensures all cells are refined consistently while still meeting the minimum required refinement. All cells in which $K n_{G L}>0.05$ are labelled as DSMC cells. This cutoff value of 0.05 for continuum breakdown has been recommended ${ }^{20,21}$ and previously validated ${ }^{13,14}$ within the hybrid code for similar flow conditions. These DSMC regions are then extended a few cells further into the continuum regions in order to create an overlap region. Particle-continuum interfaces adapt as the hybrid simulation proceeds. For future reference, the final interface locations for cases $\mathbf{M 6}_{\mathbf{R}}, \mathbf{M 1 2}_{\mathbf{R}}$, and $\mathbf{M 1 2} \mathbf{R}_{\mathbf{R}-2}$ are shown in Fig. 2. Note that cases $\mathbf{M 1 2}_{\mathbf{R}}$ and $\mathbf{M 1 2}_{\mathbf{R}-2}$ model only the boundary layer and wake regions, not the bow shock, with DSMC. Once all cell types have

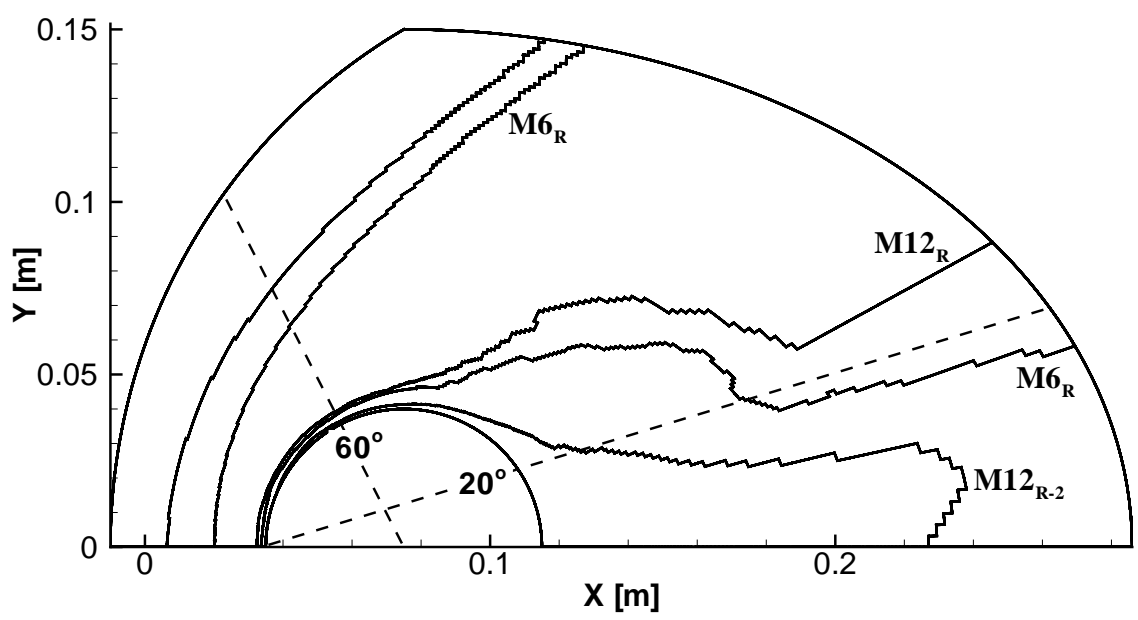

Figure 2. Final DSMC and NS domains for various flow conditions.

been defined using the initial NS solution on the continuum mesh, the refinement is carried out. For each cell labelled 
as DSMC, $r_{1} \times r_{2}$ refined cells are created and added to the DSMC mesh data structure. This creates a separate mesh refined to the mean-free-path to be used by the DSMC module. For the $\mathbf{M 6}_{\mathbf{R}}$ case, the above procedure results in the mesh shown in Fig. 3 at the start of the simulation. The mesh refinement in both the shock and wake regions is shown in Figs. 3(a) and 3(b) respectively. Although difficult to see, the refinement inside the shock varies from 6 to 12 DSMC cells for each NS cell. In the wake, as the flow expands and $\lambda$ becomes large, a corresponding reduction in the refinement level is clearly seen in Fig. 3(b). The refinement in the wake region varies from 4 cells to 1 (or no refinement).

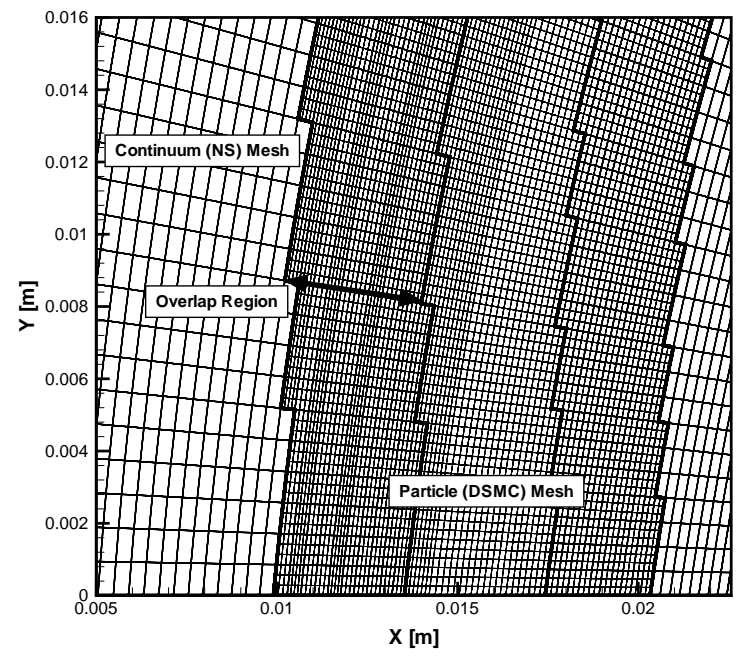

(a) Mesh refinement in the shock.

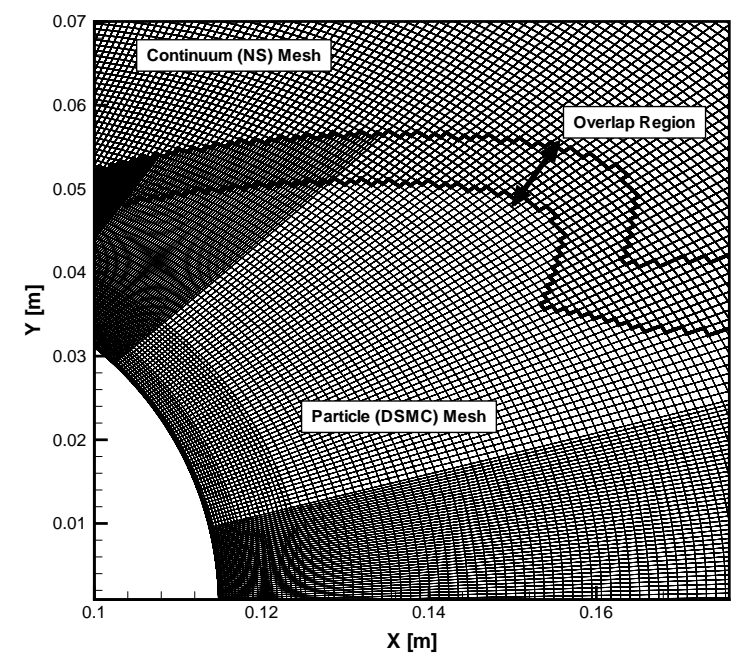

(b) Mesh refinement in the wake.

Figure 3. NS and refined DSMC meshes for case M6.

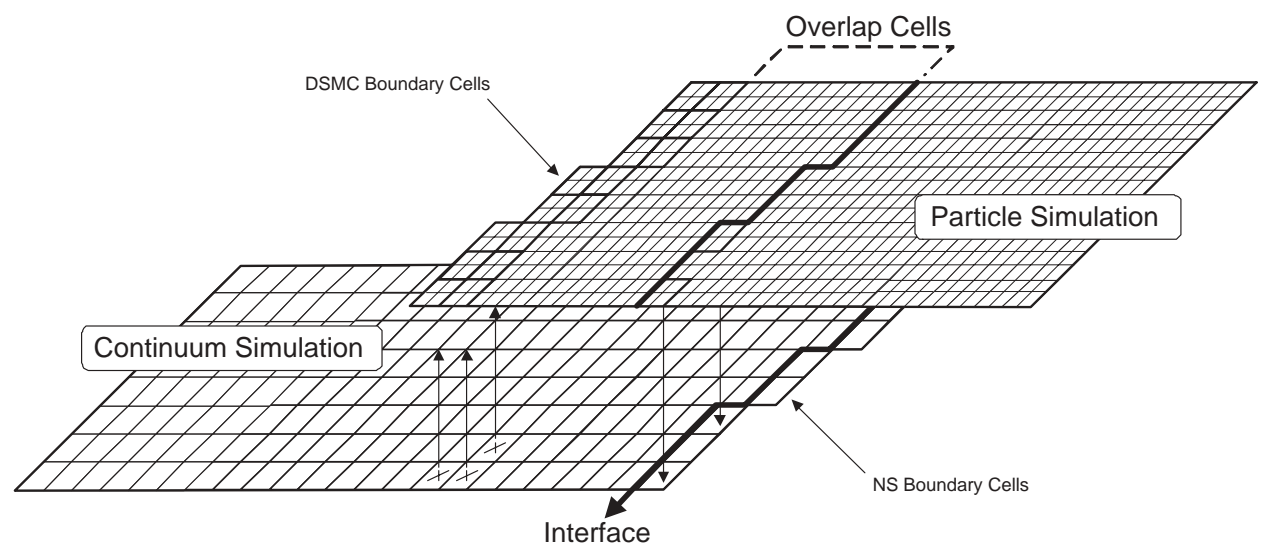

Figure 4. Information transfer and mesh refinement.

\section{B. Information Transfer}

The MPC numerical cycle, which loosely couples DSMC and NS regions while ensuring that the hybrid solution progresses towards the correct steady-state solution is entirely outlined in Ref. 14. For information transfer, the MPC method uses state-based coupling which requires only slight modification to accommodate the added mesh refinement. This method of information transfer between particle and continuum regions is depicted in Fig. 4. To summarize, on one side of the interface the state in a NS cell is used to generate a distribution of particles in the corresponding DSMC boundary cell. Particle velocities are sampled from a Chapman-Enskog distribution function. ${ }^{22}$ On the other side of 
the interface, separated by an overlap region, particle information in DSMC cells is averaged to obtain a macroscopic state which is used in the corresponding NS boundary cell. In order to reduce the statistical scatter, the sub-relaxation technique $^{23}$ which combines both spatial and temporal averaging is used to provide these macroscopic properties within each DSMC cell. As shown in Fig. 4, mesh refinement simply requires averaging over all refined DSMC cells within each NS boundary cell and also generating particles throughout multiple, refined DSMC boundary cells. The remainder of the MPC method remains un-altered. One benefit of mesh refinement is that the statistical scatter in the sub-relaxation average is reduced since larger NS cells now average over multiple DSMC cells and thus over more particles. This reduced statistical scatter results in smoother particle-continuum interfaces and also provides for smoother information transfer to the NS regions.

The fundamental mechanism that drives the hybrid solution in the proper direction is the relaxation (via collisions) of particles towards their correct non-equilibrium distributions within DSMC regions. Specifically, when a DSMC region is initialized with particles having near-equilibrium distributions consistent with a continuum solution, as particles interact and the simulation progresses in time, particle distributions (and their macroscopic representations) will progress towards the physically correct non-equilibrium solution. This behavior was first observed for 1D normal shock flows ${ }^{13}$ and later for 2D blunt-body flows. ${ }^{14}$ This progression is depicted in Fig. 5(a) where, after being initialized with particles corresponding to the NS solution, the DSMC region has iterated for 2,000 time-steps. The resultant DSMC temperature profile (calculated using the sub-relaxation technique) inside the shock for case M6 is already remarkably close to the profile determined from a full DSMC solution to the problem. This updated information in DSMC regions is then used to set boundary conditions for the NS domain. A second fundamental aspect of the MPC

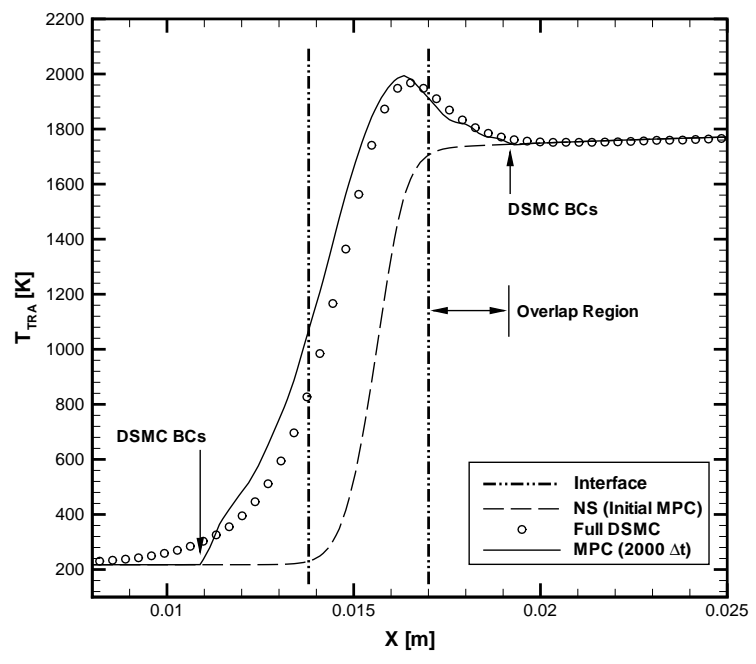

(a) Progression of the DSMC solution.

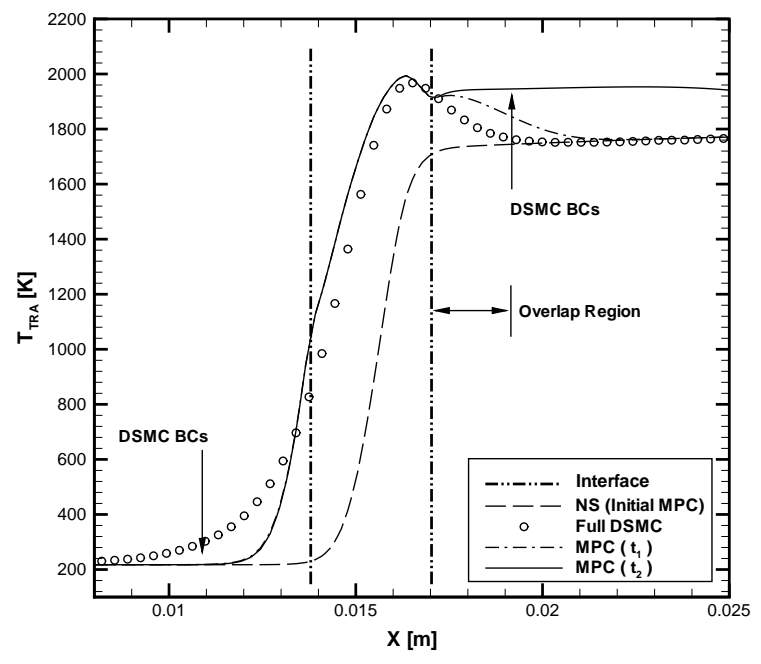

(b) Effect of mis-positioned hybrid interfaces.

Figure 5. Fundamental mechanisms of the MPC method.

method, however, is that before any information is transferred to the continuum regions, the interface must be positioned in a region accurately modelled by the NS equations. For example, if the interfaces in Fig. 5(a) remain fixed while the updated particle information is transferred into the continuum domain, significant error is introduced into the MPC simulation. This is shown in Fig. 5(b) at two times $\left(t_{1}\right.$ and $\left.t_{2}\right)$ during the NS update. Clearly the NS equations are not capable of modelling the tail of the temperature overshoot and instead simply predict a different post-shock state. This error in the NS region will then be reflected back into the particle region via the DSMC boundary cells and may never leave the simulation. Thus even if the interfaces are slightly mis-positioned, significant error can accumulate in the hybrid solution. As detailed in Ref. 14, the MPC numerical cycle utilizes the overlap region to correctly position the interfaces before any information is transferred to the continuum domain. The resulting progression/relaxation inside the DSMC regions is shown in Figs. 6(a) and 6(b) for the M6 $\mathbf{6}_{\mathbf{R}}$ case. In Fig. 6(a), the translational temperature profile through the shock wave and in the boundary is plotted as the DSMC region iterates, along the $60^{\circ}$ line shown previously in Fig. 2. Here, the MPC solution is initialized to the NS shock profile and as time increases it begins to relax towards the correct non-equilibrium temperature profile obtained from a full DSMC simulation. As the MPC solution changes, gradients spread into the overlap region which causes the interfaces to move and the DSMC region 


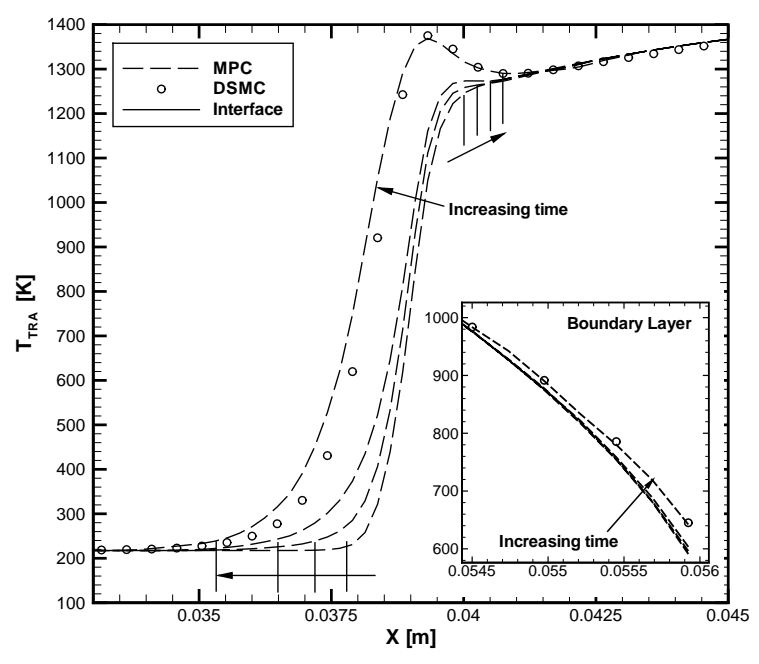

(a) Relaxation in the fore-body flow.

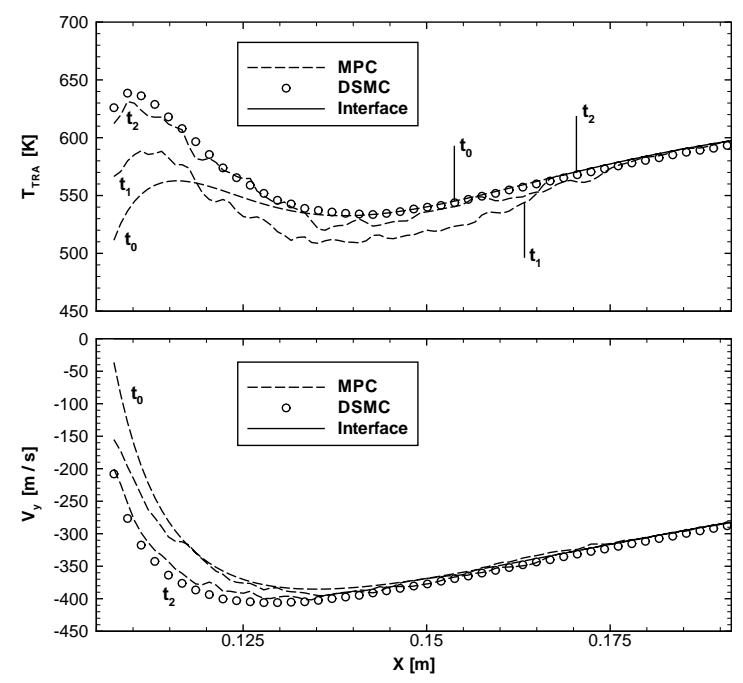

(b) Relaxation in the wake flow.

Figure 6. Relaxation from a continuum to a non-equilibrium solution.

to grow. At the same time, the temperature profile in the boundary layer also transitions from the original NS profile towards the DSMC result which is seen to have a small temperature jump at this location on the cylinder surface. This same relaxation process occurs in the near wake region and is shown in Fig. 6(b) along the $20^{\circ}$ line from Fig. 2. Here DSMC results show a much greater temperature jump and velocity slip at the wall and the MPC solution progresses significantly from the initial NS solution towards the full DSMC solution. Thus the hybrid solution progresses in the proper direction simply by allowing the DSMC simulation particles to fully relax to their non-equilibrium distributions while utilizing an overlap region to adapt the interfaces. Only when the DSMC regions and interfaces stop moving is information transferred to the NS regions which are then converged significantly on a coarser mesh with large implicit time-steps. If this updated NS solution changes the DSMC boundary conditions significantly, the process will repeat, if not, the hybrid simulation has reached a steady-state. At this point interfaces are locked in place, standard DSMC sampling begins and further convergence continues in NS regions. In this manner, a loosely coupled hybrid cycle is able to decouple the disparate temporal and spatial scales encountered in steady-state hypersonic blunt-body flows. In addition, such a loosely coupled hybrid cycle also enables the novel modular implementation described in the next paragraph.

\section{Modular Source Code and Data Structure}

The modular organization of all source code and data structures is summarized in Fig. 7. Both CFD and DSMC modules contain their original, un-modified source code (library of functions) written to operate on their own existing data structures. For example, LeMANS operates on a data structure of cells containing cell-centered information as well as on data structures containing face and node information. MONACO operates on a data structure of cells which each contain a linked-list of particles. In order to exactly retain these existing data structures, a new hybrid data structure is created to store the additional, hybrid information required by the MPC method. All hybrid information is handled at the continuum level and so for each cell in the NS mesh, a corresponding hybrid structure contains the cell's hybrid information. Such information includes the cell-type, the refinement criteria, the indices of the first and last refined DSMC cell, as well as the macroscopic, averaged properties taken over all refined DSMC cells and particles within them. For convenience, the hybrid data structure also contains a second array which matches the cell array in the DSMC module. This array contains only the indices of the larger NS cells in which the refined DSMC cells are located. This additional (and in some cases duplicate) hybrid information is found to contribute little to the overall memory requirements of the simulation. Such a modular implementation then requires few hybrid functions to both retrieve and/or modify information in either of the DSMC, NS, or hybrid data structures. For example, one hybrid function accesses the initial NS solution, calculates the continuum breakdown parameter, and sets both the cell types and the refinement criteria for each cell. A second function accesses the NS cell geometry and refinement criteria 
for each particle cell (labelled $\mathbf{P}$ in Fig. 7) and fills up the DSMC data structure with refined cells. A third function then accesses the state in each NS cell and uses it to generate particles in the corresponding refined DSMC cells. Additional hybrid functions handle information transfer by simply retrieving data from one data structure, processing it, and inserting it into the other data structure at appropriate times during the simulation. The way in which each module operates on this data remains completely un-changed. All source code libraries are linked such that hybrid

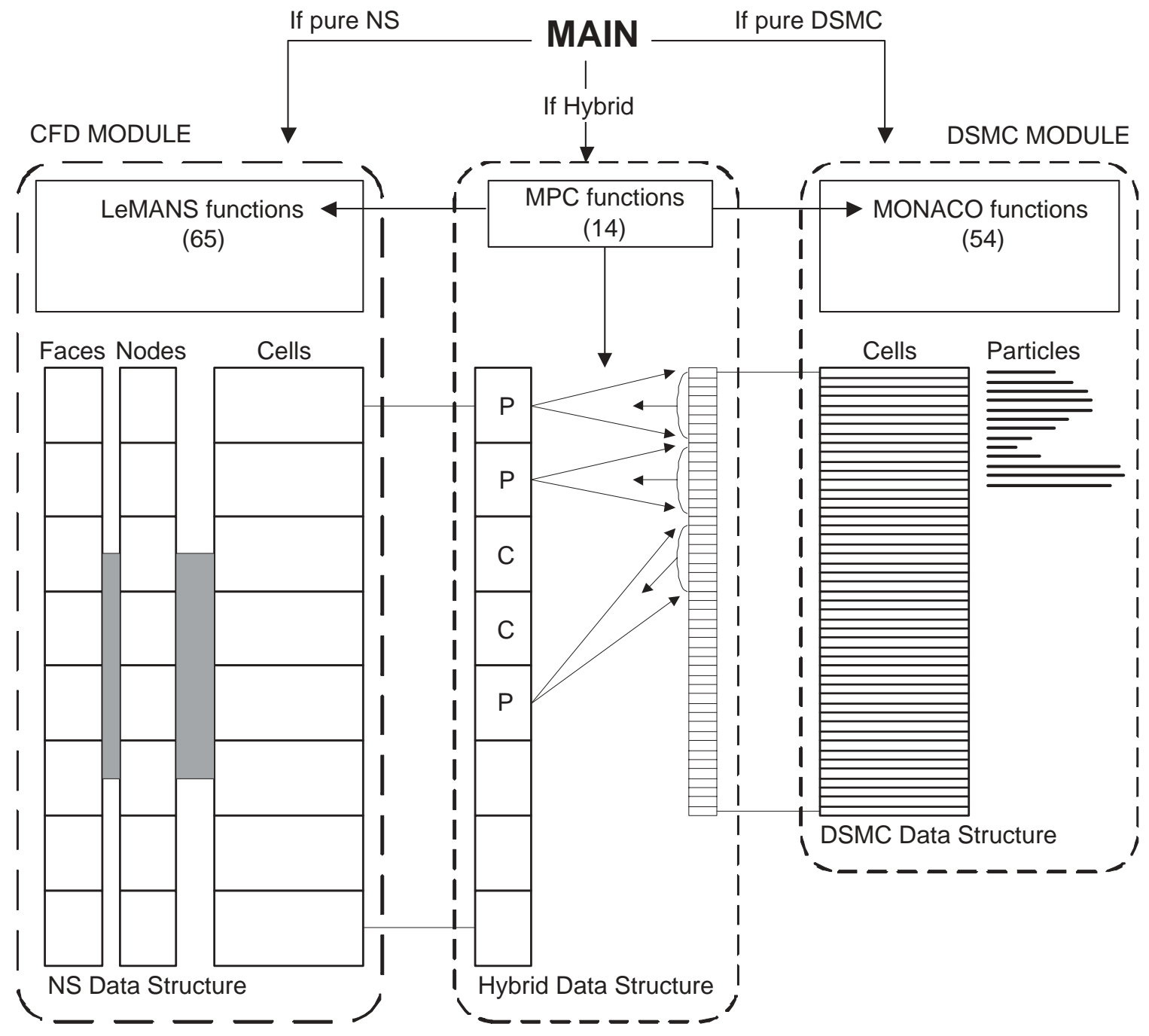

Figure 7. Modular organization of source code and data structures.

functions have access to all functions from both the DSMC and NS modules and thus have access to all information at all times. The original main functions from both DSMC and NS modules are kept available using compiler flags so that a user may run the simulation in either pure DSMC (MONACO) or pure NS (LeMANS) mode. When running in hybrid (MONACO-LeMANS) mode, the main function consists of a mixture of DSMC, NS, and new hybrid functions which implement the MPC numerical cycle. ${ }^{14}$ Since high-level DSMC and NS functions can be used as-is, currently only 14 new hybrid functions are required, 4 of which are solely devoted to input and output of simulation data. These functions must of course be written specifically to interface with the exiting DSMC and NS data structures. However, if significant updates are made to either module, the complete source code of a new version can be inserted, un-modified. It is only the hybrid functions that may require modification in order to remain consistent. In the current architecture, since the entire NS mesh is stored, the one exception is added logic in the LeMANS source code ensuring the NS module only operates on cells labelled as continuum cells. This modular implementation employed by the MPC method has potential benefits for multi-scale and multi-disciplinary engineering simulations. 


\section{Numerical Results and Performance}

The accuracy of the MPC method using mesh refinement (cases $\mathbf{M} \mathbf{3}_{\mathbf{R}}, \mathbf{M} \mathbf{6}_{\mathbf{R}}$, and $\mathbf{M} \mathbf{1 2} \mathbf{2}_{\mathbf{R}}$ ) is similar to the accuracy obtained without mesh refinement (cases M3, M6, and M12, reported in Ref. 14). It should be noted that since case $\mathbf{M 1 2}_{\mathbf{R}}$ does not model the shock wave using DSMC, it corresponds to case M12a from Ref. 14. The largest discrepancy between MPC and full DSMC simulations is found in continuum regions where the NS equations model the gas with a single temperature and thus do not capture thermal non-equilibrium effects. However, close to the cylinder surface where the hybrid code uses the DSMC method, the results agree very well. For example, Fig. 8(a)

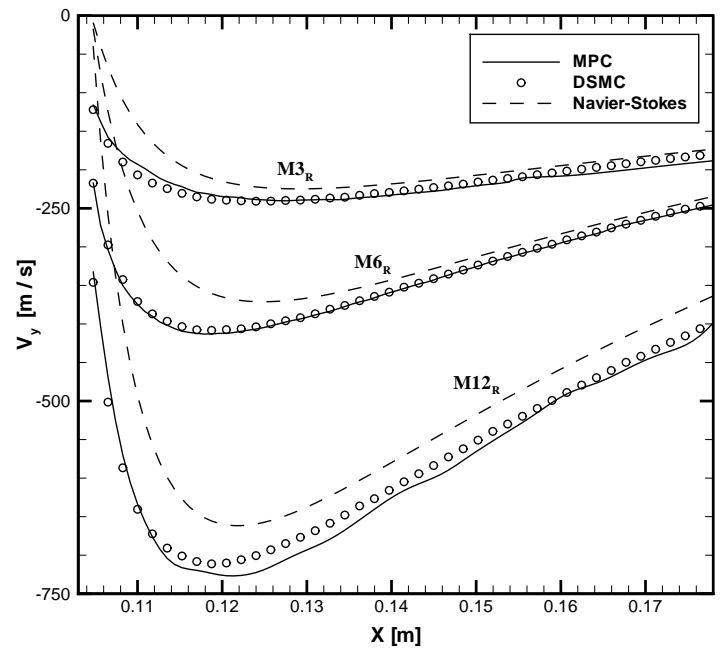

(a) $y$-velocity profiles.

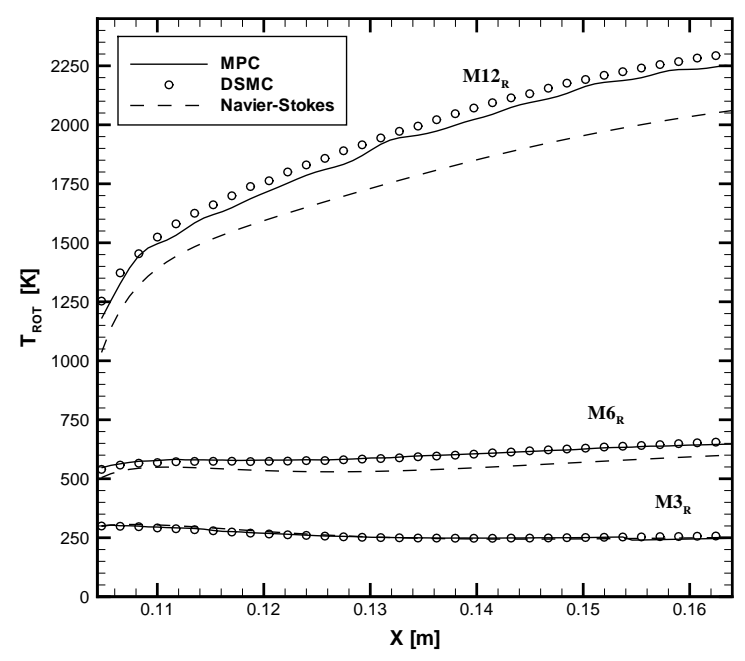

(b) Rotational temperature profiles.

Figure 8. Simulation results in the near-wake region (transitional flow).

shows the resulting $y$-velocity profiles for cases $\mathbf{M} \mathbf{3}_{\mathbf{R}}, \mathbf{M} \mathbf{6}_{\mathbf{R}}$, and $\mathbf{M} \mathbf{1}_{\mathbf{R}}$, along the $20^{\circ}$ line shown previously in Fig. 2. In this highly non-equilibrium region of the near-wake, significant velocity slip is evident in the DSMC results, which is captured accurately by the MPC method. Figure 8(b) plots the rotational temperature along the same $20^{\circ}$ line for each case. Here, the MPC method is also seen to accurately reproduce the rotational temperature calculated from full DSMC simulations in the near-wake region. Thus, although the MPC code is not capable of modelling thermal nonequilibrium in NS regions of the hybrid simulation, it is able to accurately model the rotational energy mode inside the DSMC regions. It is further determined that the largest discrepancy between previous results without mesh refinement and the current results using mesh refinement is found in the bow shock region of the flow. In contrast to previous results,${ }^{14}$ the hybrid particle-continuum interfaces are now determined on a coarse NS mesh. On this mesh, the shock wave may only span a few cells and thus a difference of even one cell in the location of the interface can result in the shock position calculated by the MPC method to be shifted by a noticeable amount. This can be seen for the $\mathbf{M 6}_{\mathbf{R}}$ case in Fig. 6(a), presented earlier in this article, where the MPC temperature-shock profile is seen to sit slightly upstream of the profile computed by a full DSMC simulation. Thus precise modelling of the shock wave location and interior using the MPC method likely requires an initial NS mesh and solution which uses more cells to resolve the bow shock region. However, as Fig. 8 shows for the $\mathbf{M 1 2}_{\mathbf{R}}$ case, and as previous results have shown, ${ }^{14}$ precise resolution of the shock wave interior is not necessary for accurate prediction of the flow conditions near the cylinder body.

The added mesh refinement now enables the calculation of lower Knudsen number flows where the mean-free-path outside of the near-wake (especially in the stagnation region) becomes extremely small and thus full DSMC simulation becomes extremely computationally demanding. To test the MPC code, the free-stream density for case $\mathbf{M 1 2}_{\mathbf{R}}$ was raised by a factor of five, resulting in case $\mathbf{M 1 2}_{\mathbf{R}-2}$ having a global Knudsen number of 0.002 . A portion of the refined mesh near the top of the cylinder is shown in Fig. 9. The final interface location for this case was shown previously in Fig. 2 where the non-equilibrium region simulated using DSMC is seen to be much smaller than for case $\mathbf{M 1 2}_{\mathbf{R}}$. Specifically, the entire fore-body and most of the wake is deemed by the breakdown parameter to be continuum flow. The solution obtained by a full NS simulation is verified to match the solution of a full DSMC simulation in the forebody region, however, the results differ slightly in the near-wake region. The full DSMC simulation was obtained on 


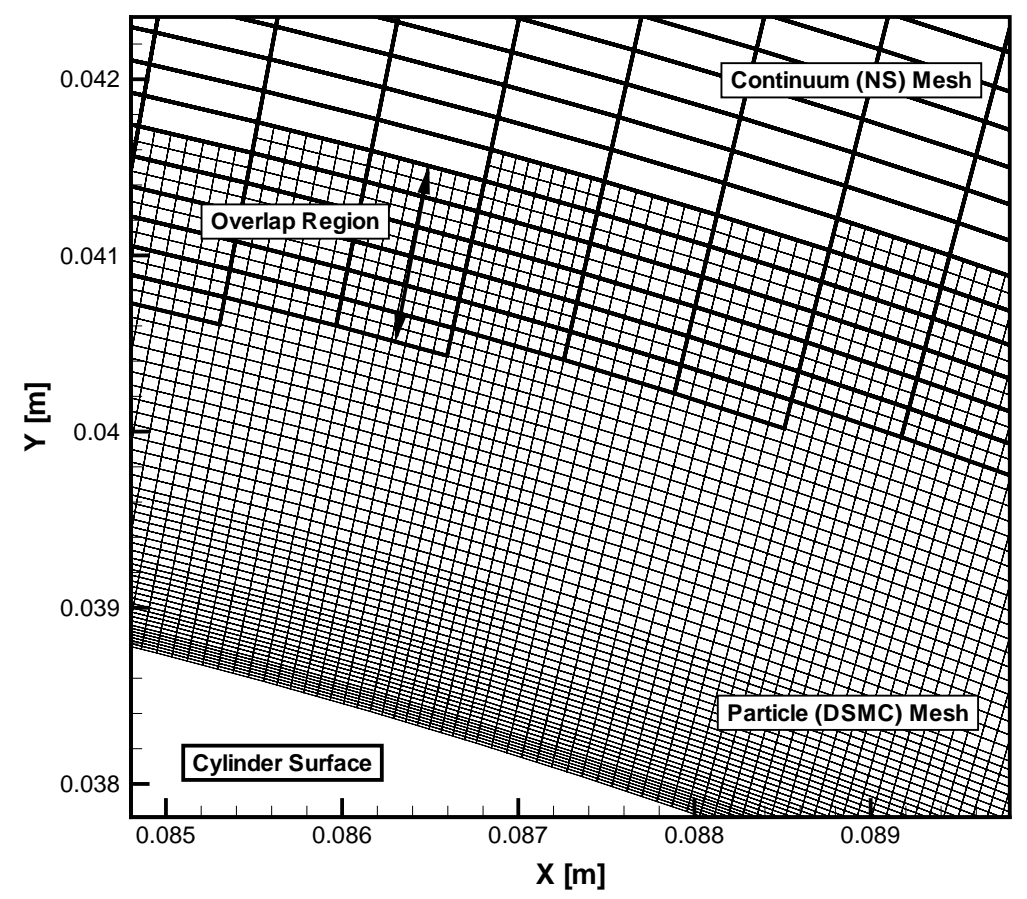

Figure 9. Refined mesh for case $\mathrm{M} 12_{\mathrm{R}-2}$.

an unstructured mesh adapted locally between $2 \lambda$ and $3 \lambda$, and used the sub-cell technique to ensure that collision cells were no larger than $\lambda$. The resulting simulation contained 570,000 cells, 110 million particles, and used a constant time-step of $4 \times 10^{-9} s$. The results of the MPC simulation for case $\mathbf{M 1 2}_{\mathbf{R}-\mathbf{2}}$ are compared with this full DSMC result in addition to a pure NS result in Fig. 10. To be consistent with the full DSMC simulation, the MPC mesh was refined using $F=2$ in Eq. 4 and the sub-cell technique was used in the DSMC module. Figure 10(a) plots both translational and rotational temperatures along the $20^{\circ}$ line (from Fig. 2) revealing the degree of thermal non-equilibrium in the near-wake region. This phenomenon is predicted accurately by the MPC method near the surface where the DSMC method is used, however is not captured on the other side of the interface where the NS equations assume a single temperature. This level of discrepancy between MPC and full DSMC results is un-avoidable unless the rotational energy mode is separately modelled within the continuum module or unless hybrid-DSMC regions are enlarged to contain all regions of thermal non-equilibrium. However, for blunt-body flows, the latter would result in the majority of the flow being modelled using DSMC. Since accurate modelling of the flow near the surface is of more practical importance, this discrepancy in continuum regions (away from the surface) may be acceptable. The accuracy of the MPC method close to the cylinder surface is verified in greater detail in Fig. 10(b). Here, the temperatures (top) and $y$-velocity (bottom) are plotted at a distance of $8 \times 10^{-4} \mathrm{~m}$ (or $0.2 \%$ of the cylinder radius) from the surface in the wake region $\left(90^{\circ}<\theta<180^{\circ}\right)$. The MPC method is seen to improve upon the initial NS solution and accurately reproduce full DSMC results. The gas is seen to be near thermal equilibrium at $90^{\circ}$, exhibit noticeable thermal non-equilibrium effects while expanding around the cylinder, and finally return to thermal equilibrium during re-compression at the base of the cylinder $\left(180^{\circ}\right)$. Similarly, the $y$-velocity profile obtained by DSMC agrees well with the NS profile near the top and base of the cylinder, however differs significantly from the NS solution in between where the flow is rapidly expanding. In this region, where significant velocity slip is present, the MPC method successfully reproduces the DSMC velocity profile. However, the top of Fig. 10(b) also highlights a substantial error (3.2\%) in the temperatures predicted by the MPC method near the top of the cylinder $\left(90^{\circ}\right)$. Case $\mathbf{M 1 2}_{\mathbf{R}-2}$ is the first MPC simulation where the particle-continuum interface actually intersects the cylinder surface and, as seen in Fig. 2, the interface remains close to the surface of the cylinder up to the $120^{\circ}$ point. The precise explanation for the temperature discrepancy in this region of the hybrid mesh has not yet been investigated but may be a consequence of the small number of DSMC cells between the interface and the cylinder surface. 


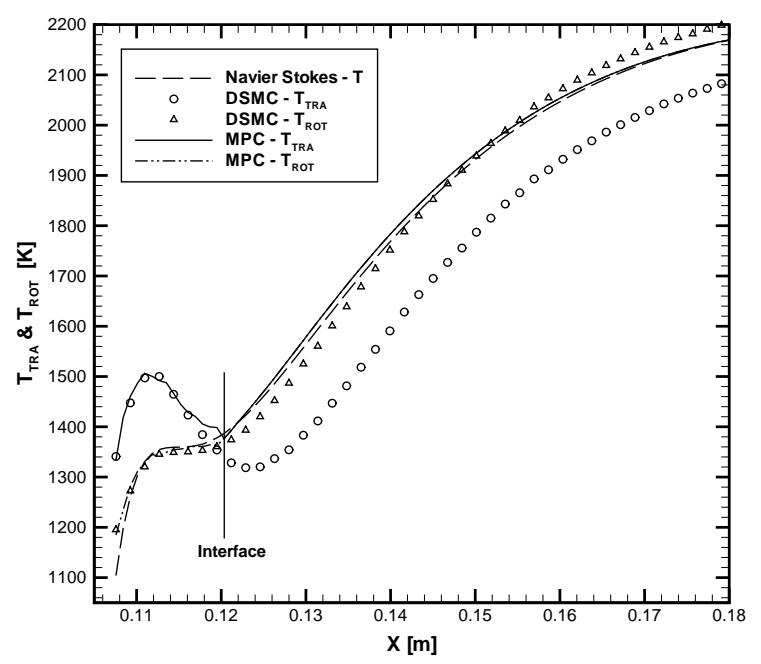

(a) Temperature profiles in the cylinder wake.
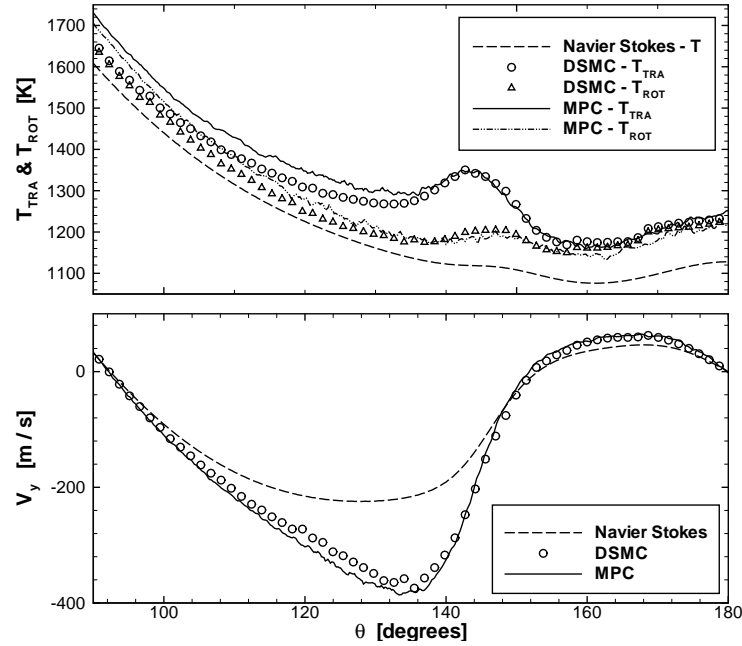

(b) Flow properties along the cylinder surface.

Figure 10. Simulation results in the near-wake region (mainly continuum flow).

In limiting the DSMC method only to regions of non-equilibrium flow, the MPC method reproduces full DSMC results while using a fraction of the particles. The addition of mesh refinement to the MPC method reduces its memory requirements and enables the NS module to operate efficiently on a coarser mesh. The NS equations are solved using a point implicit method with a CFL number of 10. This results in continuum time-steps ranging from 20-25 times larger for the transitional flows and 92 times larger for the mainly continuum flow compared to the time-steps used in the corresponding full DSMC simulations. The decrease in both overhead and memory requirements of the MPC method achieved by mesh refinement are detailed in Table 1. The data for cases M3, M6, and M12 is taken from Ref. 14. Here, the actual speedup is simply the ratio of time required for full DSMC simulation to that required by a MPC simulation. In order to make a fair comparison, after reaching steady-state, both MPC and full DSMC simulations are sampled for the same number of time-steps. A hybrid simulation samples fewer particles than a full DSMC simulation, however must also update the NS regions periodically. The ideal speedup is simply equal to the ratio of particles required for a full DSMC simulation to the number required by the MPC simulation. Since the time required for a full DSMC simulation scales linearly with the total number of particles, if all hybrid operations were negligible, the largest possible (or ideal) speedup would be equal to this ratio. The MPC overhead is given by the difference between ideal and actual speedup factors divided by the ideal speedup. The memory usage is the ratio of memory used by a MPC simulation to that required by a full DSMC simulation. Table 1 shows that added mesh refinement has cut the MPC overhead roughly in half for each case. This is entirely due to the decrease in time required for both the initial NS solution and for NS updates of the continuum regions during the MPC numerical cycle. For

Table 1. Computational performance and memory requirements of the MPC method.

\begin{tabular}{|c||c|c|c|c|}
\hline case & actual speedup & ideal speedup & MPC overhead & memory usage \\
\hline \hline M6 & 1.62 & 2.37 & $31.6 \%$ & $137 \%$ \\
\hline M6 $_{\mathbf{R}}$ & 2.10 & 2.58 & $18.6 \%$ & $69 \%$ \\
\hline \hline M3 & 2.24 & 2.98 & $24.8 \%$ & $134 \%$ \\
\hline M3 $_{\mathbf{R}}$ & 2.73 & 3.11 & $12.2 \%$ & $51 \%$ \\
\hline \hline M12 & 2.75 & 4.10 & $33.0 \%$ & $97 \%$ \\
\hline $\mathbf{M 1 2}_{\mathbf{R}}$ & 3.27 & 3.80 & $14.0 \%$ & $32 \%$ \\
\hline \hline $\mathbf{M 1 2}_{\mathbf{R}-2}$ & 10.6 & 11.15 & $5.0 \%$ & $8 \%$ \\
\hline
\end{tabular}


the flow conditions used in cases $\mathbf{M} \mathbf{3}_{\mathbf{R}}, \mathbf{M} \mathbf{6}_{\mathbf{R}}$, and $\mathbf{M} \mathbf{1}_{\mathbf{R}}$, the speedup factors achieved with mesh refinement are now $2.73,2.10$, and 3.27 respectively. Since previous results without mesh refinement stored the same mesh (refined to the mean-free-path) in both NS and DSMC modules, as Table 1 shows, the MPC simulations actually required more memory than full DSMC simulations despite using fewer particles. The addition of mesh refinement has substantially lowered the memory requirements for the NS module. As a result, MPC simulations now require less memory than full DSMC simulations. Since the non-equilibrium region for case $\mathbf{M 1 2}_{\mathbf{R}-\mathbf{2}}$ is highly localized, the MPC method uses 11.15 times fewer particles than a corresponding full DSMC simulation and requires only $8 \%$ of the memory. The MPC results for case $\mathbf{M 1 2}_{\mathbf{R}-\mathbf{2}}$ presented in the previous paragraph were obtained 10.6 times faster than the corresponding full DSMC simulation and the MPC overhead is measured to be only 5\%. It is important to note that the full DSMC simulation is obtained on a parallel computer, whereas the MPC solution for case $\mathbf{M 1 2}_{\mathbf{R}-\mathbf{2}}$ is obtained on a PC. Due to parallel efficiency, and different processor speeds, precise calculation of the relative performance is difficult and the values listed in Table 1 for this case are approximate. As a result, the overhead value of 5\% is somewhat questionable. However, in general, as the number of particles increases in a MPC simulation, the overhead is expected to diminish (especially that associated with the initial NS solution and NS updates).

\section{Conclusions}

The implementation of a modular particle-continuum (MPC) numerical method is described and tested on simple blunt-body flows under transitional and mainly continuum conditions. A mesh refinement procedure is outlined which uses a structured mesh designed for the continuum equations in NS regions and refines this mesh to the local mean-free-path for use by the DSMC method in non-equilibrium regions. As a result, the MPC method is able to accurately resolve the non-equilibrium regions using the computationally intensive DSMC method while solving the NS equations in continuum regions on a coarser mesh using larger implicit time-steps. In this manner the MPC method is able to effectively decouple the disparate length and time scales found in hypersonic flows. In addition, a novel modular implementation accommodated by the MPC method is detailed. This implementation allows for existing, state-of-the-art, DSMC and NS source codes to be incorporated virtually un-modified within the hybrid code. Such an implementation reduces both the development time of a hybrid code as well as the time required to incorporate new versions of either module. Previous baseline hybrid simulations of Mach 3, 6, and 12 transitional flow about a 2D cylinder are revisited using the added mesh refinement capability. The MPC simulation accuracy is maintained while mesh refinement cuts the overhead inherent in the MPC method in half. As a result, the MPC method reproduces full DSMC solutions using 51\%, 69\%, and 32\% of the memory required by DSMC and obtaining the solution 2.73, 2.1, and 3.27 times faster than DSMC for the Mach 3, 6, and 12 flows respectively. The MPC method with added mesh refinement is further tested for Mach 12 flow over a cylinder at a higher density where the global Knudsen number of the flow is 0.002. In this mainly continuum flow, non-equilibrium effects are localized within the near-wake region of the cylinder only. The MPC method uses 11.2 times fewer particles than a full DSMC simulation and obtains the solution approximately 10.6 times faster while using only $8 \%$ of the memory required by a full DSMC simulation. MPC results agree well with full DSMC results which differ from a NS solution only in the near-wake region of the flow. The largest discrepancy between MPC and full DSMC simulations is found away from the cylinder surface where hybrid-NS regions do not account for thermal non-equilibrium in the expanding flow. The largest discrepancy near the surface occurs wherever the hybrid interface closely approaches or intersects the cylinder surface itself, and is an issue which requires future analysis.

\section{Acknowledgments}

This work is sponsored by the Space Vehicle Technology Institute, under NASA grant NCC3-989 with joint sponsorship from the Department of Defense and from the Air Force Office of Scientific Research grant FA9550-05-1-0115. This work is also supported by the Francois-Xavier Bagnoud Foundation.

\section{References}

\footnotetext{
${ }^{1}$ Bird, G. A., Molecular Gas Dynamics and the Direct Simulation of Gas Flows, Oxford University Press, New York, 1994.

${ }^{2}$ Moss, J. N. and Price, J. M., "Survey of Blunt Body Flows Including Wakes at Hypersonic Low-Density Conditions," Journal of Thermophysics and Heat Transfer, Vol. 11, No. 3, 1997, pp. 321-329.

${ }^{3}$ Wilmoth, R. G., Mitcheltree, R. A., Moss, J. N., and Dogra, V. K., "Zonally Decoupled Direct Simulation Monte Carlo Solutions of Hypersonic Blunt-Body Wake Flows," Journal of Spacecraft and Rockets, Vol. 31, No. 6, 1994, pp. 971-979.
} 
${ }^{4}$ Glass, C. E. and Gnoffo, P. A., “A 3-D Coupled CFD-DSMC Solution Method With Application to the Mars Sample Return Orbiter,” NASA TM-2000-210322, July 2000.

${ }^{5}$ Glass, C. E. and Horvath, T. J., "Comparison of a 3-D CFD-DSMC Solution Methodology With a Wind Tunnel Experiment," NASA TM2002-211777, Aug. 2002.

${ }^{6}$ Wright, M., Prabhu, D., and Martinez, E., "Analysis of Apollo Command Module Afterbody Heating Part I: AS-202," Journal of Thermophysics and Heat Transfer, Vol. 20, No. 1, 2006, pp. 16-30.

${ }^{7}$ Hash, D. B. and Hassan, H. A., "Assessment of Schemes for Coupling Monte Carlo and Navier-Stokes Solution Methods," Journal of Thermophysics and Heat Transfer, Vol. 10, No. 2, 1996, pp. 242-249.

${ }^{8}$ Hash, D. B. and Hassan, H. A., "A Decoupled DSMC/Navier-Stokes Analysis of a Transitional Flow Experiment," AIAA Paper 96-0353, Jan. 1996, presented at the 34th AIAA Aerospace Sciences Meeting and Exhibit, Reno, NV.

${ }^{9}$ Roveda, R., Goldstein, D. B., and Varghese, P. L., "Hybrid Euler/Particle Approach for Continuum/Rarefied Flows," Journal of Spacecraft and Rockets, Vol. 35, No. 3, 1998, pp. 258-265.

${ }^{10}$ Roveda, R., Goldstein, D. B., and Varghese, P. L., "Hybrid Euler/Direct Simulation Monte Carlo Calculation of Unsteady Slit Flow," Journal of Spacecraft and Rockets, Vol. 37, No. 6, 2000, pp. 753-760.

${ }^{11}$ Wijesinghe, H. S., Hornung, R. D., Garcia, A. L., and Hadjiconstantinou, N. G., "Three-dimensional Hybrid Continuum-Atomistic Simulations For Multiscale Hydrodynamics," Journal of Fluids Engineering, Vol. 126, 2004, pp. 768-777.

${ }^{12}$ Wijesinghe, H. S. and Hadjiconstantinou, N. G., "A Discussion of Hybrid Atomistic-Continuum Methods for Multiscale Hydrodynamics," International Journal for Multiscale Computational Engineering, Vol. 2, 2004.

${ }^{13}$ Schwartzentruber, T. E. and Boyd, I. D., "A hybrid particle-continuum method applied to shock waves," Journal of Computational Physics, Vol. 215, 2006, pp. 402-416.

${ }^{14}$ Schwartzentruber, T. E., Scalabrin, L. C., and Boyd, I. D., "Hybrid Particle-Continuum Simulations of Non-Equilibrium Hypersonic Blunt Body Flow Fields," AIAA Paper 06-3602, June 2006, presented at the 9th AIAA Joint Thermophysics and Heat Transfer Conference, San Francisco, CA.

${ }^{15}$ Dietrich, S. and Boyd, I. D., "Scalar and Parallel Optimized Implementation of the Direct Simulation Monte Carlo Method," Journal of Computational Physics, Vol. 126, 1996, pp. 328-342.

${ }^{16}$ Boyd, I. D., "Analysis of Rotational Nonequilibrium in Standing Shock Waves of Nitrogen," AIAA Journal, Vol. 28, No. 11, 1990, pp. 19971999.

${ }^{17}$ Scalabrin, L. C. and Boyd, I. D., "Development of an Unstructured Navier-Stokes Solver for Hypersonic Nonequilibrium Aerothermodynamics," AIAA Paper 05-5203, 2005, presented at the 38th AIAA Thermophysics Conference, Toronto, Ontario, Canada.

${ }^{18}$ MacCormack, R. W. and Candler, G. V., "The Solution of the Navier-Stokes Equations Using Gauss-Seidel Line Relaxation," Computers and Fluids, Vol. 17, 1989, pp. 135-150.

${ }^{19}$ Gnoffo, P. and White, J., “Computational Aerothermodynamic Simulation Issues on Unstructured Grids,” AIAA Paper 04-2371, June 2004, presented at the 37th AIAA Thermophysics Conference, Portland, OR.

${ }^{20}$ Boyd, I. D., Chen, G., and Candler, G. V., "Predicting Failure of the Continuum Fluid Equations in Transitional Hypersonic Flows," Physics of Fluids, Vol. 7, No. 1, 1995, pp. 210-219.

${ }^{21}$ Wang, W.-L. and Boyd, I. D., "Predicting Continuum Breakdown in Hypersonic Viscous Flows," Physics of Fluids, Vol. 15, 2003, pp. 91100 .

${ }^{22}$ Garcia, A. L. and Alder, B. J., "Generation of the Chapman-Enskog Distribution,” Journal of Computational Physics, Vol. 140, 1998, pp. 66-70.

${ }^{23}$ Sun, Q. and Boyd, I. D., "Evaluation of Macroscopic Properties in the Direct Simulation Monte Carlo Method," Journal of Thermophysics and Heat Transfer, Vol. 19, No. 3, 2005, pp. 329-335. 